\title{
Racism and the empathy for pain on our skin
}

\section{Matteo Forgiarini* , Marcello Gallucci* and Angelo Maravita}

Department of Psychology, University of Milano-Bicocca, Milan, Italy

Edited by:

Nadia Bolognini, University of

Milano-Bicocca, Italy

Reviewed by:

Nadia Bolognini, University of

Milano-Bicocca, Italy

Marco Perugini, University of

Milano-Bicocca, Italy

*Correspondence:

Marcello Gallucci and Matteo Forgiarini

Faculty of Psychology, Milan Bicocca

University, Piazza dell'Ateneo Nuovo,

1-20126 Milano, Italy.

e-mail:marcello.gallucci@unimib.it;

e-mail: matteo.forgiarini@gmail.com
Empathy is a critical function regulating human social life. In particular, empathy for pain is a source of deep emotional feelings and a strong trigger of pro-social behavior. We investigated the existence of a racial bias in the emotional reaction to other people's pain and its link with implicit racist biases. Measuring participants' physiological arousal, we found that Caucasian observers reacted to pain suffered by African people significantly less than to pain of Caucasian people. The reduced reaction to the pain of African individuals was also correlated with the observers' individual implicit race bias. The role of others' race in moderating empathic reactions is a crucial clue for understanding to what extent social interactions, and possibly integration, may be influenced by deeply rooted automatic and uncontrollable responses.

Keywords: racism, empathy, prejudice, pain, skin conductance

\section{INTRODUCTION}

Empathy is the ability to understand and vicariously share the feelings and thoughts of other people (De Vignemont and Singer, 2006). Empathic feelings are fundamental for humans in social and interpersonal life because they enable human beings to tune their mental states to their social environment as well as to understand others' intentions, actions, and behaviors. One of the main sources of empathic feelings is the pain experienced by other human beings, and empathy for others' pain, in turn, regulates behavior among individuals and social groups. Although pain has been considered an intimate and private feeling, experimental data indicate that when people witness or imagine the pain of another person, they map the others' pain onto their brain using the same network activated during firsthand experience of pain, as if they were vicariously experiencing the observed pain (Hutchison et al., 1999; Carr et al., 2003; Wicker et al., 2003; Morrison et al., 2004; Singer et al., 2004; Bufalari et al., 2007; Lamm et al., 2007; Cheng et al., 2008). Furthermore feeling other people's suffering triggers pro-social behavior (Batson et al., 2002), promotes helping and encourages cooperation (Batson et al., 1997a). Conversely, lack of empathy for the pain of other human beings may lead to violence, abuse, and deterioration of interpersonal and intergroup relationships (Batson et al., 2002).

Empathic reactions to pain involve different layers of cognitive processing, with a predominant role played by automatic and implicit processes. Recent neurophysiological findings (Singer et al., 2004) have documented specific neuropsychological activations of the affective but not sensory components of the brain (the pain matrix in particular), leading to fast and automatic responses to the pain of others. Similarly, the vision of a needle penetrating the hand reduces the muscular motor response in the observer compatible with the locus of injection in the target person (Avenanti et al., 2005). Immediate empathic reactions, however, are deeply affected by social cues and individual differences. We now know that functional activity related to empathy reactions to others' feelings is affected by similarity between the witness and the person in pain (Krebs, 1975; Preston and de Waal, 2001; Lamm et al., 2010), by previous experience in the same situation (Cheng et al., 2007), by observer's personality (Avenanti et al., 2009), by social relationship with the target (Singer et al., 2006), by familiarity for the target (Cialdini et al., 1997), gender (Eisenberg and Carlo, 1995; Baron-Cohen and Wheelwright, 2004), and age (Eisenberg and Morris, 2001). Most relevant for the present work, initial findings seem to suggest that empathy for others pain can be modulated by ingroup/outgroup social categorization based on race differences between the target and the onlooker (Cosmides et al., 2003; Xu et al., 2009; Avenanti et al., 2010).

If immediate empathic reactions differ depending on the race of the target person, this modulation may be rooted in personal characteristics of the person experiencing empathy, allowing for individual differences in the strength of the link between race and empathy. A weaker reaction to pain of other race members may be a consequence of personal prejudices and, more generally, racist attitudes toward the outgroup member. It is unlikely, however, that immediate physiological reactions are modulated by explicit and elaborated cognitive processes. We reckon that the possible link between racist attitudes and weaker empathy reactions for others' pain should operate at an implicit level, where prejudices and racism manifest themselves through fast and unconscious associations between negative evaluations and the target race (Greenwald et al., 1998). Along this line of reasoning, a recent independent TMS study (Avenanti et al., 2010) using a Blacks/Whites measure of implicit racist attitude (Greenwald et al., 1998), showed that despite the lack of explicit racial bias in the sample, participants with higher implicit ingroup preference presented greater corticospinal reactivity to ingroup models over outgroup models' pain.

The present research is aimed at providing experimental evidence that automatic, physiological reactions to other people's pain strongly depends on the race of the person in pain, such that pain received by members of other racial groups elicits a much weaker reaction compared with the pain suffered by members of the same group. By presenting participants with a series of video clips, in two experiments we tested whether the reaction to pain of Caucasian (Italian) observers was influenced by the race (Caucasian, Asian, 
or African) of the person in pain. In the second study we replicate this finding and show that the moderation of empathy is correlated with the individual implicit racial biases.

Empathic reactions were inferred by the skin conductance responses (SCR; Purves et al., 2008) to observed video stimuli showing human subjects experiencing either harmless or painful somatosensory stimuli. The third-person exposure to pain activates a brain network called "pain matrix" (Peyron et al., 1999, 2000; Derbyshire, 2000) which includes the anterior cingulate cortex. Activity in the ACC is known to trigger variation in the skin conductance (SC; Purves et al., 2008), i.e., SC increases as a physiologic and autonomic response to someone else's pain (Krebs, 1975; Levenson and Ruef, 1992; Morrison et al., 2004; Rae Westbury and Neumann, 2008). The triggering role of the ACC on SC is likely to have been selected to facilitate coping and adaptive responses (Devinsky et al., 1995; Ledowski et al., 2006). Furthermore, SC is considered one of the most reliable predictors of accurately assessed negative emotions in others, as it is associated with the emotional response rather than the mere pain recognition (Levenson and Ruef, 1992).

For the above reasons, the SCR was chosen as a likely marker of the automatic, emphatic response to the vision of painful stimuli inflicted in another person.

\section{EXPERIMENT 1}

\section{MATERIALS AND METHODS}

Stimuli were video clips showing a person subject to a painful stimulus or a harmless one. Each video started with a frame depicting a face of a female or a male actor holding a neutral expression. Subsequently the camera zoomed in on the actor's hand which was touched by the experimenter alternatively by an eraser (harmless stimulus) or by a needle (painful stimulus). A total of 12 video clips were presented to each participant, featuring six different actors: Two Caucasian, two African, and two Asian actors. For each race, a female and a male actor was used, each actor subject to a painful stimulus and a harmless one.

In both experiments, participants sat in front of a computer monitor (Acer aspire 1360, monitor 15.4" TFT LCD) where the experimental stimuli were displayed. The distance between the monitor and participant's face was almost $70 \mathrm{~cm}$. Prior to stimulus presentation, two electrodes were applied on the forefinger and ring-finger of participant's left hand in order to record the SCR. Participants were asked to relax, and carefully watch the stimuli presented on the monitor. During SCR recording, participants were listening to white noise with headphones in order to cover external auditory stimuli. The videos order was completely randomized. The experimenter, blind to stimuli presentation, started each video after visually checking that the online SCR was returned to a baseline level, in order to avoid response overlaps to consecutive stimuli. Following this procedure the inter stimulus interval was $15 \mathrm{~s}$ (range 10-20) across all participants. Each experimental session lasted almost $45 \mathrm{~min}$, including behavioral and psychophysiological data gathering. All participants gave their consents to physiological recording and display of videos prior to the experiments. After the experiments ended, participants were fully debriefed regarding the nature of the stimuli and aim of the study.

Skin conductance responses was measured while the observers viewed the video stimuli. The difference between the SCR subsequent to a painful stimulus and the SCR subsequent to a harmless stimulus was taken as a measure of empathy for pain (hereinafter empathic index, EI). Physiological data collection was performed using The UFI model 2701 BioDerm(R) SC meter. It is a stand-alone instrument which measures skin conductance level (SCL) and SCR. Skin conductance is measured using an $\mathrm{Ag}-\mathrm{AgCl}$ electrode pair with the constant voltage $(0.5 \mathrm{~V})$ method. The SCR were recorded as the phase component of the SC activity, with a $10-\mathrm{Hz}$ rate. Microsiemens ( $\mu \mathrm{s})$ are the measurement units. For all the participants, the SCR recordings were synchronized with the first video frame presented. The average response within a time window of $6.5 \mathrm{~s}$ post-stimulus was used as the observed variable for the analysis ${ }^{1}$. This interval was chosen based on the relevant literature (Purves et al., 2008) as the most appropriate and included the whole variation of SC following the stimuli. The SCR data analysis was performed using the SAS General Linear Model procedure. Unless otherwise specified, all the results discussed were obtained with a least squares repeated-measures ANOVA. The estimated means for the stimulus $\times$ race interaction at different levels (1 SD below and 1 SD above the mean) of the continuous implicit association test (IAT) variable were obtained with an equivalent model estimated with the PROC MIXED SAS procedure (i.e., SAS procedure commonly used to estimate mixed effects linear models).

Ninety students of Milano-Bicocca University have been recruited. Three participants were excluded for problems in data saving; 5 participants were excluded because of uncooperative behavior during the experiment; 17 participants were excluded due to technical problems during the experiment. Out of the 65 remaining participants, four were excluded as outliers: SCR scores exceeding 2 SD from the overall average. A total of 61 participants (29 female) were therefore included in the analyses.

Participants were subjected to a 2 (stimuli: harmless and painful $) \times 3$ (races of the target person: African, Caucasian, Asian $) \times 2$ (blocks: first and second experimental block) $\times 2$ (target gender: male and female) repeated-measure factorial design.

\section{RESULTS}

Participants showed an overall significant EI: reactions to painful stimuli were significantly greater than reactions to harmless stimuli $[F(1,59)=40.85, P<0.001]$.

Crucially, the race of the actor experiencing the painful stimulus significantly moderated the EI $[F(2,118)=3.6, P=0.03]$. Although experimental participants showed a significant EI for Caucasians $\left[F(1,59)=29.57, P<0.001, \eta^{2}=0.333\right]$, for Africans $[F(1,59)=7.52$, $\left.P=0.008, \eta^{2}=0.113\right]$, and for Asian images $[F(1,59)=16.99$, $\left.P<0.001, \eta^{2}=0.223\right]$, the empathic reaction for the Caucasians was significantly greater than that for the Africans $[F(1,59)=7.87$, $P=0.006, \eta^{2}=0.117$; Figure 1 $]$. Critically, there was no racial effect on the reaction to the harmless stimuli $[F(2,118)=0.09, P=0.91]$, a significant moderating effect of target person race was found on the reaction to painful stimuli $[F(2,118)=5.09, P=0.007]$ : Reactions to Caucasians painful stimuli were significantly greater than for Africans $[t(118)=2.91, P=0.004]$ but not than for Asian targets $[t(118)=1.72, P=0.08]$.

${ }^{1}$ This type of analysis is consistent with technical manual published by the producer of the UFI BioDerm System (http://www.ufiservingscience.com/). 
As expected, the SCR responses significantly varied during the time for the painful stimuli, but not for the harmless stimuli. Specifically, the reactions to painful stimuli significantly reduced over time [block effect: $F(1,59)=44.58, P<0.0001]$ and the reactions to harmless stimuli were constant during the experiments [block effect: $F(1,59)=0.15, P=0.70]$. No other effect was statistically significant.

\section{EXPERIMENT 2: IMPLICIT ATTITUDES AND EMPATHY FOR PAIN \\ MATERIALS AND METHODS}

The second experiment aimed at linking the empathic racial bias with the implicit racial prejudice. In addition to using the same paradigm used in Experiment 1, the experiment required participants to complete a race (Caucasians/Africans) IAT (Greenwald et al., 1998) and a Trait Empathy Scale (Mehrabian and Epstein, 1972). Specifically, we assessed to what extent individual differences in the implicit racial prejudice correlate with the difference between the EI for Caucasians with respect to EI for Africans.

The IAT provides access to deep cognitive domains that is not reached by self-report measures (Greenwald et al., 1998). In the version used in this study, it provides a measure of implicit differential evaluation of Caucasian and African races. The IAT is based on participants' reaction times on a computer-based categorization task. The IAT assesses the association between two classes of stimuli by measuring differences in the response speed that participants show in the same task with exemplars from two categories. The task we used rates the association strength between positive and negative concepts with Caucasian and African races. On each trial of the race IAT we used, participants categorized a stimulus from one of four the categories: a photo of a Caucasian man, a photo a African man, a positive word (Joy, love, peace, wonderful pleasure, friend), or a negative word (agony, terrible, awful, bad, evil, war). In one block of trials, positive words required the same behavioral response as photos of Caucasian men. In another block of trials, positive words required the same response as photos of African men. IAT data were coded in the direction of association between positive words and Caucasian targets, i.e., as the difference in mean response latency

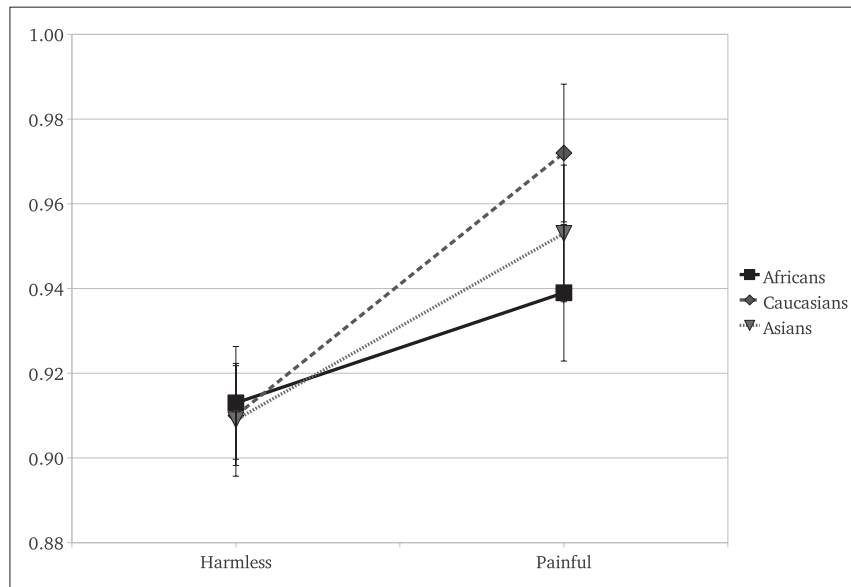

FIGURE 1 | Experiment 1: mean SCR and standard errors as a function of stimulus type and actor's race. Responses to pain were always greater than those to the harmless stimuli. to trials in positive word-African targets block minus trials in the positive words-Caucasian targets block. Then, higher scores reflect strong associations between positive concepts and Caucasian race as well as strong association between negative concepts and African race. The fourth and seventh blocks consisted of 40 trials, all of the other blocks consisted of 20 trials. IAT scores reported in the text and used in the analysis are D scores. We calculated D scores using Nosek's SAS macro based on Greenwald et al. (2003). Individual IAT scores were used in the mixed model as a continuous independent variable. The simple slopes analysis was conducted to estimate the experimental effects at specific values of the IAT score. This was obtained by centering the IAT scores to 1 SD above the mean (and subsequently at $1 \mathrm{SD}$ below) before entering the IAT score in the model (Aiken and West, 1991).

The experiment included 60 students of Milano-Bicocca University. Two participants were excluded for problems in data saving; five participants were excluded due to technical problems during the experiment. Out of these 53 participants, 6 were excluded as outliers (SCR scores exceeding 2 SD from overall average). A total of 47 participants ( 24 female) were therefore included in the analyses.

\section{RESULTS}

Results replicated the overall stronger reaction to painful than to harmless stimuli $[F(1,45)=36.63, P<0.001]$. Target race significantly moderated the EI $[F(2,90)=4.26, P=0.01$; Figure 2]. The EI was significant for Caucasian $[F(1,45)=23.85, P<0.0001$, $\left.\eta^{2}=0.346\right]$ and Asian $\left[F(1,45)=13.9, P=0.0005, \eta^{2}=0.225\right]$ but not for African actors $\left[F(1,45)=1.36, P=0.24, \eta^{2}=0.029\right]$. Furthermore, the EI was statistically lower for Africans than for Caucasians $[F(1,45)=6.64, P=0.01]$ targets (Figure 2). As for Experiment 1, we observed no race differences in reactions for harmless stimuli $[F(2,90)=0.1, P=0.9]$ and a race significant effect on the reaction for other people's pain $[F(2,90)=7.55, P<0.001]$. The SCR and the IAT scores were analyzed together. In the IAT used, greater scores indicate faster associations of "Caucasians" with positive concepts and "Africans" with negative concepts, thus a

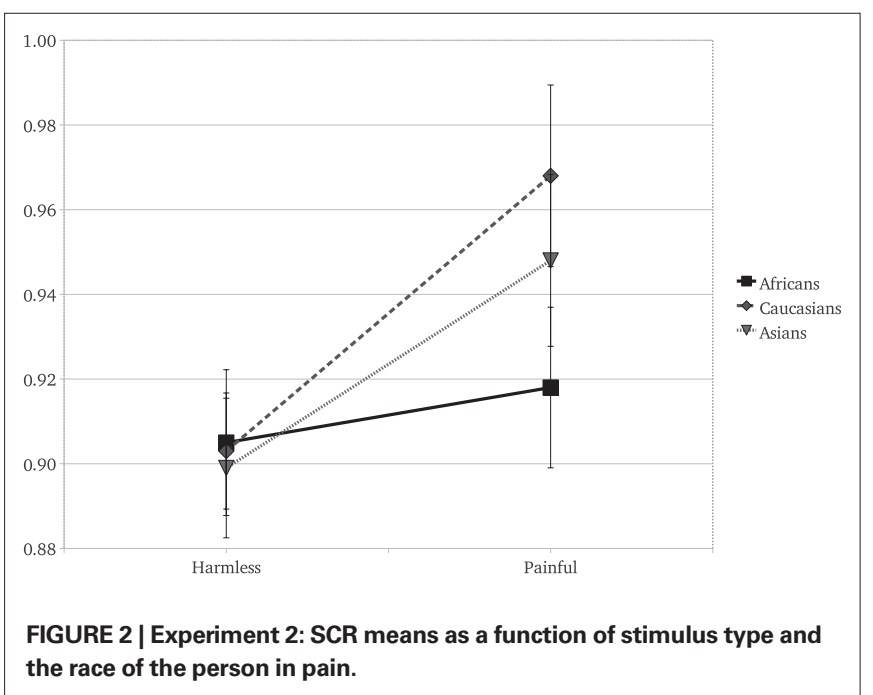


stronger racial bias against "Africans." The IAT scores were included in a GLM comprising the SCR values elicited only by Africans and Caucasians stimuli (the two races included in the IAT). The IAT scores were included in the model as a continuous independent variable. As typically found with the race IAT, Caucasian observers more strongly associated negative stereotypes with Africans than with Caucasians, $[F(1,46)=34.45, P<0.001]$. Most importantly, the strength of the implicit race bias correlates with the reduced empathy for Africans' pain. We found that the IAT scores of the observers significantly predict the moderating effect of race on the reaction for pain $[F(1,43)=4.52, P=0.03]$. Simple slope analysis (Aiken and West, 1991) revealed that the greater the participant racial bias, the greater the difference between the empathic responses toward Caucasians with respect to Africans (Figure 3). Data show that participants with low race bias (1 SD below sample average) are not affected by the race moderating effect on the empathic responses to actors' pain (Figure 3B). Participants EI is significantly greater than zero $[F(1,45)=5.22, P=0.02]$ but it's not moderated by the race of the person in pain $[F(1,45)=0.14$, $P=0.70]$ and there are no differences in the overall reactions for Caucasians and Africans $[F(1,45)=1.28, P=0.25]$. On the other hand, data show that for participants with an high race bias (IAT score $1 \mathrm{SD}$ above the sample average) EI is significant greater than zero $[F(1,45)=14.52, P=0.0001]$ and the race of the person in pain significantly moderates the empathic reactions $[F(1,45)=13.29$, $P=0.0003$; cf. Figure 3A].

The BEES empathy scale completed by participants had no significant effects on the SCR EI for any of three races we tested. Even though the BEES seems to account for the empathy related brain areas activation (Singer et al., 2004), this empathy scale seems to failed in prediction of SCR empathy related activations (Rae Westbury and Neumann, 2008).

As regards effects over time, reactions to painful stimuli significantly reduced over time [block effect: $F(1,45)=8.08, P=0.006$ ] and the reactions to harmless stimuli were constant during the experiments [block effect: $F(1,45)=1.67, P=0.20$ ]. These results replicated Experiment 1 results, suggesting that participants' stimuli perception were reliable and precise during the entire experiment. No other effect was significant.
In order to rule out possible alternative explanations, we performed the analysis of SCR baseline values immediately before participants' empathic reactions. The mean of SCR values during the 600-ms prestimulus was calculated. As expected, in Experiments 1 and 2 the full model revealed no relevant effects for all the experimental factors on the pre-stimulus SCR. These findings rule out the possibility that the observed responses associated with the painful and harmless stimuli were due to stochastic effects prior to stimulus presentation.

\section{EXPERIMENTS 1 AND 2: GENERAL DISCUSSION}

Taken together our findings demonstrate a clear pattern of responses to pain: the extent to which Caucasian observers share the pain experience of other people is affected by the race of the person in pain (Figure 4A). Before the stimulus onset, the SCR values show stochastic variations. After observing a painful stimulus administered to the target person, participants' SCR values increase more for Caucasian targets than for target people of the other races, and the least for African targets.

This race moderation pattern was not present in the reactions to harmless stimuli (Figure 4B). During the video stimuli perception, before and after the stimulus onset, participants' responses are not affected by the race of the target people.

Given the link between automatic SCR response and emotional response to an observed situation, our data suggest that implicit and uncontrolled cognitive mechanisms lead Caucasians to reduce the automatic sharing of pain experience with African conspecifics at an automatic, early level of stimulus processing. These data concur with studies demonstrating deep connections between implicit social evaluations and neurological responses of the central nervous system. Research in the field of social neuroscience shows that in white participants the strength of amygdale activation to unfamiliar black vs. white faces is correlated with implicit but not explicit race bias measures (Phelps et al., 2000) and is related to different level of PFC activity (Cunningham et al., 2004).

The correlation between the empathic reactions as measured with the SCR and the IAT scores rules out alternative explanations of the effect based on some low-level perceptual features of the video stimuli presented. In fact, perceptual artifacts should have a general reduction of response to African actors for both harmless and painful stimuli,

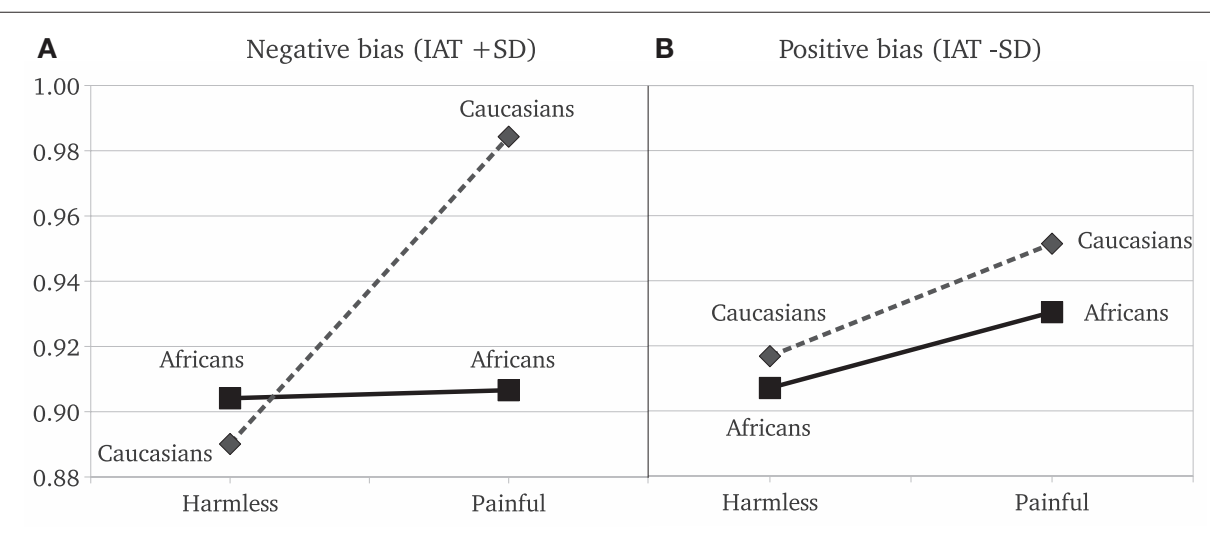

FIGURE 3 | Experiment 2: simple slope analysis. Estimated SCR means as a function of stimulus type and the race of the person computed at two different levels of IAT scores: (A) estimation for strong negative bias for Blacks (1 SD above average IAT score); (B) estimation for positive bias for Blacks (1 SD below average IAT score). 

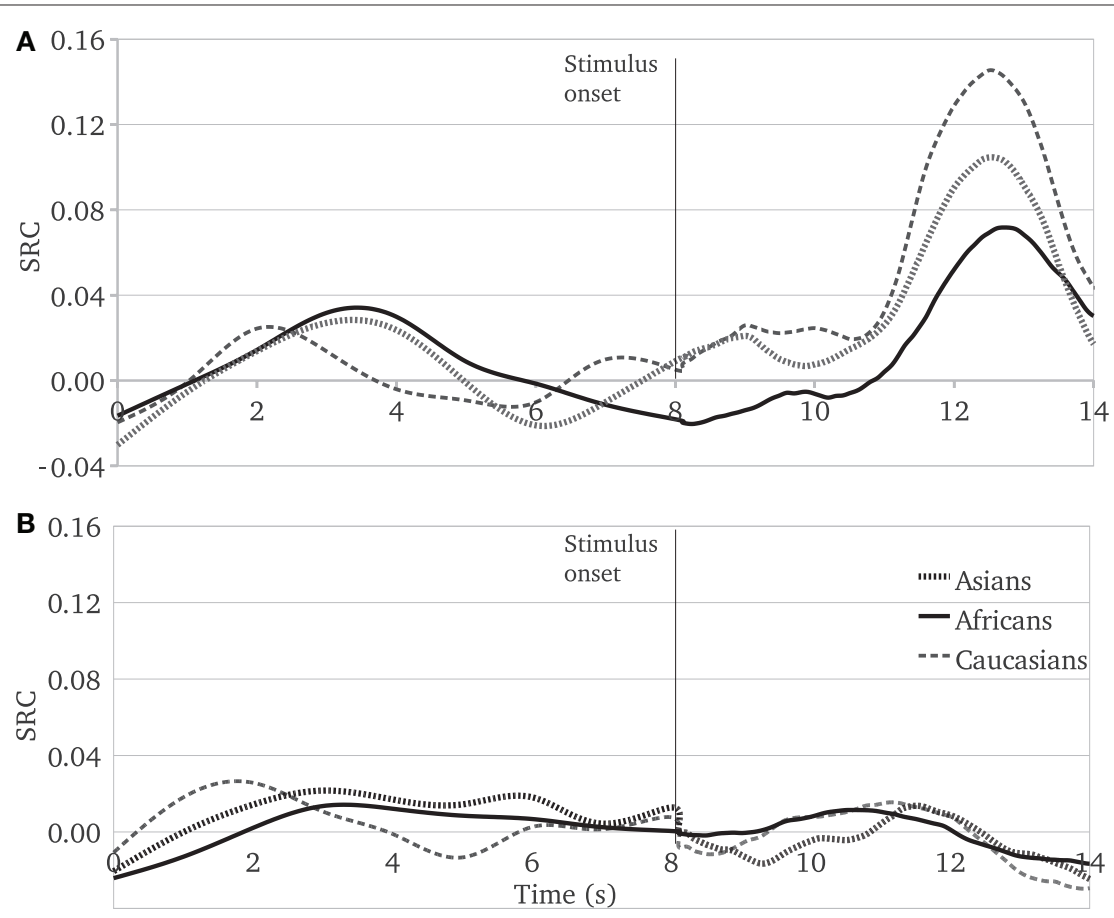

FIGURE 4 | Experiments 1 and 2, SCR mean values as a function of time and race of target people. (A) Reactions to painful stimuli, (B) Reactions to harmless stimuli.

whereas the moderation due to race is specifically found for painful stimuli ${ }^{2}$. As our data did not show any gender effect, mere similarity between actors and observers could not account for our results.

Interestingly, these data do not support the outgroup antipathy hypothesis (Brown et al., 2006) as they do not indicate increased affective reactions to stimuli of outgroup members in general. Moreover, the ingroup empathy hypothesis (Brown et al., 2006) does not seem to account for our effects either. A mere ingroupoutgroup categorization should lead to a significant reduction of empathy for Africans as well as Asian actors. Instead, the EI to Africans was lower than the one for Caucasians and Asian targets in both experiments. Furthermore, although one of the most pervasive categorizations in human society is gender, we never observed an interaction between the subject's gender and stimulus gender in the empathic responses (in both of the Experiments 1 and 2, Ps > 0.63). Interestingly findings regarding a differential reaction to animals in pain (Rae Westbury and Neumann, 2008) suggest that empathic feelings in humans are moderated by the perceived phylogenetic similarity between the observer and the suffering animal. In a similar vein, data gathered in the two studies, seem to indicate that the closer the phenotypic aspect of the actor and the observer, the stronger the psychophysiologic empathic response to pain. Consistently with our data Batson et al. (1997b) found that university group membership (i.e., shared or unshared) had no impact on empathetic induction.

${ }^{2}$ Although our results are clear and in line with the theoretical expectations, further research is needed to understand the extent to which the effects we found could be replicated using different sets of stimuli and different physiological markers.
Conversely, Johnson et al. (2002) showed that the defendant race clearly affected the empathetic induction on a subsequent juror decision-making task. One reason for the divergent findings might involve the differential nature of the groups studied. One obvious explanation is that race might be more relevant or salient than university membership (Cunningham, 1986; Krebs, 1991). In a similar vein, Cosmides et al. (2003) noted that racial group membership defines coalitions and alliances during evolution and thus results in strong modulation of the neural substrates of emotional components of empathy.

Thus, previous research and our findings suggest that relevant group membership might play a significant role in empathetic induction. But how can people rate differences of race? And, what does really means being members of two different races? Since it is very difficult to directly measure the degree of genetic similarity with others, Krebs (1991) has suggested that one relies on discernible cues to make such judgments. Clearly, racial indicators (e.g., skin color, hair texture) would seem to qualify as powerful cues of kinship and genetic similarity. Furthermore recent findings showed that racial biases affect clinical pain management: Pletcher et al. (2008) provided evidence that physicians withhold opioid treatment from Hispanic, Black, and Asian patients compared to White patients, despite similar pain severity. The authors also noted this therapeutic disparity cannot be attributed to patient histories of alcohol and drug abuse as disproportionate treatment was most apparent in patients under the age of 12 .

Moreover, our data support the idea that racial groups different from the perceiver could elicit a weaker sense of familiarity than a more similar conspecific. Dehumanization Theory (Fiske 
et al., 2002) posits that some specific social groups are perceived as less human as they activate non-exclusively social emotions. The so called Stereotyped Content Model predicts that perceived outgroups' warmth and competence affect what emotions outgroups elicit in perceivers. Our data are consistent with this model and findings we reported seem to place race beside warmth and competence as a predictor of shared emotions. According to Singer's theory phenotypically distant outgroups may even elicit different patterns of brain activation, with particular regard to the medial PFC (Harris and Fiske, 2006), a region of cortex implicated in social cognition. This region responds to faces of people belonging to all social groups except extreme outgroups who activate, instead, a pattern consistent with disgust. Different degrees of dehumanization may therefore account for our findings that Caucasians could perceive Asians and Africans on different levels of humanization. Our findings indicate that even if we are not aware, our body and our mind use internalized knowledge to address reactions and activities they engage to deal with social and physical world. Future research may strengthen the link between Dehumanization Theory and differential empathic reactions to pain.

Clearly, relationship between race and empathy has many significant implications for real-world issues. Would racial differences decrease the probability that a Black teacher could empathize with a White student? Would racial similarity increase the probability that a White supervisor could empathize with a White subordinate? How a manager choose the person who has to be fired between a

\section{REFERENCES}

Aiken, L. S., and West, S. G. (1991). Multiple Regression: Testing and Interpreting Interactions. Thousand Oaks: Sage.

Avenanti, A., Bueti, D., Galati, G., and Aglioti, S. M. (2005). Transcranial magnetic stimulation highlights the sensorimotor side of empathy for pain. Nat. Neurosci. 8, 955-960.

Avenanti, A., Minio-Paluello, I., Bufalari, I., and Aglioti, S. M. (2009). The pain of a model in the personality of an onlooker: influence of state-reactivity and personality traits on embodied empathy for pain. Neuroimage 44, 275-283.

Avenanti, A., Sirigu, A., and Aglioti, S. M. (2010). Racial bias reduces empathic sensorimotor resonance with otherrace pain. Curr. Biol. 20, 1018-1022.

Baron-Cohen, S., and Wheelwright, S. (2004). The empathy quotient: an investigation of adults with Asperger syndrome or high functioning autism, and normal sex differences. J. Autism Dev. Disord. 34, 163-175.

Batson, C. D., Chang, J., Orr, R., and Rowland, J. (2002). Empathy, attitudes and action: can feeling for a member of a stigmatized group motivate one to help the group. Pers. Soc. Psychol. Bull. 28, 1656-1666.

Batson, C. D., Polycarpou, M. P., HarmonJones, E., Imhoff, H. J., Mitchener, E.

Black and a White worker? Although the teacher, the supervisor and the manager might make an effort to empathize, they are likely to be biased and experience less empathy for Blacks involved in their choices.

Our findings suggest that the attitude toward other races may involve not only the overt self-report of the observer concerning attitudes about race but also their deep automatic and physiological reactions. These differential reactions may be elicited even at a very basic level, such as the reaction to physical pain of others. Such a fundamental racial differentiation, in turn, may bias complex activities and judgments over and beyond human consciousness. A precise assessment of other people's pain, in fact, is a necessary skill in many human activities, from medical decisions, rescue operations, police intervention, policy making and, in extreme circumstances, use of physical force and punishment. When all these activities involve people perceived as belonging to different races, a racial bias may hinder pain assessment with detrimental effects on individuals, groups, and their peaceful relationships.

\section{ACKNOWLEDGMENTS}

This work was supported by funding from the University of Milano-Bicocca (FAR) to Marcello Gallucci and Angelo Maravita. Preliminary data were presented to the General Meeting of the European Association of Experimental Social Psychology, 10-14 June 2008, Opatija, Croatia.

cephalography study. Neuroimage 40 1833-1840.

Cialdini, R. B., Brown, S. L., Lewis, B. P., Luce, C., and Neuberg, S. L. (1997) Reinterpreting the empathy-altruism relationship: when one into one equals oneness. J. Pers. Soc. Psychol. $73,481-494$.

Cosmides, L., Tooby, J., and Kurzban, R. (2003). Perceptions of race. Trends Cogn. Sci. (Regul. Ed.) 7, 173-179.

Cunningham, M. (1986). Levites and brother's keepers: a sociobiological perspective on prosocial behavior. Humboldt J. Soc. Relat. 13, 35-67.

Cunningham, W. A., Johnson, M. K., Raye, C. L., Gatenby, J. C., Gore, J. C., and Banaji, M. R. (2004). Separable neural components in the processing of black and white faces. Psychol. Sci. 15 806-813.

De Vignemont, F., and Singer, T. (2006). The empathic brain: how, when and why? Trends Cogn. Sci. 10, 435-441.

Derbyshire, S. (2000). Exploring the pain "neuromatrix". Curr. Pain Headache Rep. 4, 467-477.

Devinsky, O., Morrell, M. J., and Vogt, B. A. (1995). Contributions of anterior cingulate cortex to behaviour. Brain $118,279-306$.

Eisenberg, N., and Carlo, G. (1995). Prosocial development in late adolescence: a longitudinal study. Child Dev. 66, 1179-1197.
Eisenberg, N., and Morris, A. S. (2001). The origins and social significance of empathy-related responding. A review of empathy and moral development: implications for caring and justice by M. L. Hoffman. Soc. Justice Res. 14, 95-120.

Fiske, S. T., Cuddy, A. J., Glick, P., and Xu, J. (2002). A model of (often mixed) stereotype content: competence and warmth respectively follow from perceived status and competition. J. Pers. Soc. Psychol. 82, 878-902.

Greenwald, A., McGhee, D. E., and Schwartz, J. L. K. (1998). Measuring individual differences in implicit cognition: the implicit association test. J. Pers. Soc. Psychol. 74, 1464-1480.

Greenwald, A. G., Nosek, B. A., and Banaji, M. R. (2003). Understanding and using the implicit association test: I. An improved scoring algorithm. J. Pers. Soc. Psychol. 85, 197-216.

Harris, L. T., and Fiske, S. T. (2006). Dehumanizing the lowest of the low: neuroimaging responses to extreme out-groups. Psychol. Sci. 17, 847-853.

Hutchison, W. D., Davis, K. D., Lozano, A. M., Tasker, R. R., and Dostrovsky, J. O. (1999). Pain-related neurons in the human cingulate cortex. Nat. Neurosci. 2, 403-405.

Johnson, J., Simmons, C., Jordan, A., MacLean, L., Taddei, J., and Thomas, 
D. (2002). Rodney King and O. J. revisited: the impact of race and defendant empathy induction on judicial decisions. J. Appl. Soc. Psychol. 32, 1208-1223.

Krebs, D. (1975). Empathy and altruism. J. Pers. Soc. Psychol., 32, 1134-1146.

Krebs, D. L. (1991). Altruism and egoism: a false dichotomy. Psychol. Inq. 2, 137-139.

Lamm, C., Batson, C. D., and Decety, J. (2007). The neural substrate of human empathy: effects of perspective-taking and cognitive appraisal. J. Cogn. Neurosci. 19, 42-58.

Lamm, C., Meltzoff, A. N., and Decety, J. (2010). How do we empathize with someone who is not like us? A functional magnetic resonance imaging study. J. Cogn. Neurosci. 22, 362-376.

Ledowski, T., Bromilow, J., Paech, M. J., Storm, H., Hacking, R., and Schug, S. A. (2006). Monitoring of skin conductance to assess postoperative pain intensity. Br. J. Anaesth. 97, 862-865.

Levenson, R. W., and Ruef, A. M. (1992). Empathy: a physiological substrate. J. Pers. Soc. Psychol. 63, 234-246.
Mehrabian, A., and Epstein, N. (1972). A measure of emotional empathy. J. Pers. 40, 525-543.

Morrison, I., Lloyd, D., Di Pellegrino, G., and Roberts, N. (2004). Vicarious responses to pain in anterior cingulate cortex: is empathy a multisensory issue? Cogn. Affect. Behav. Neurosci. 4, 270-278.

Peyron, R., Garcia-Larrea, L., Gregoire, M.-C., Costes, N., Convers, P., Lavenne, F., Mauguiere, F., Michel, D., and Laurent, B. (1999). Haemodynamic brain responses to acute pain in humans: sensory and attentional networks. Brain 122, 1765-1780.

Peyron, R., Laurent, B., and García-Larrea, L. (2000). Functional imaging of brain responses to pain. A review and metaanalysis (2000). Neurophysiol. Clin. 30, 263-288.

Phelps,E.A.,O'Connor, K. J., Cunningham, W. A., Funayama, E. S., Gatenby, J. C., Gore, J. C., and Banaji, M. R. (2000). Performance on indirect measures of race evaluation predicts amygdala activation. J. Cogn. Neurosci. 12, 729-738.

Pletcher, M. J., Kertesz, S. G., Kohn, M. A., and Gonzales, R. (2008). Trends in opioid prescribing by race/ethnicity for patients seeking care in US emergency departments. JAMA 299, 70-78.

Preston, S. D., and de Waal, F. B. M. (2001). Empathy: its ultimate and proximate bases. Behav. Brain Sci. 25, 1-20.

Purves, D., Brannon, E. M., Cabeza, R. Huettel, S. A., LaBar, K. S., Platt, M. L. and Woldorff, M. G. (2008). Principles of Cognitive Neuroscience. Sunderland, MA: Sinauer Associates Inc.

Rae Westbury, H., and Neumann, D. L. (2008). Empathy-related responses to moving film stimuli depicting human and non-human animal targets in negative circumstances. Biol. Psychol. 78, 66-74.

Singer, T., Seymour, B., O’Doherty, J. Kaube, H., Dolan, R. J., and Frith, C. D. (2004). Empathy for pain involves the affective but not sensory components of pain. Science 303, 1157-1162.

Singer, T., Seymour, B., O’Doherty, J. P., Stephan, K. E., Dolan, R. J., and Frith, C. D. (2006). Empathic neural responses are modulated by the perceived fairness of others. Nature 439 466-469.

Wicker, B., Keysers, C., Plailly, J., Royet, J.-P., Gallese, V., and Rizzolatti, G. (2003). Both of us disgusted in my insula: the common neural basis of seeing and feeling disgust. Neuron 40, 655-664.

Xu,X.,Zuo,X., Wang,X., and Han, S. (2009). Doyou feel my pain? Racial group membership modulates empathic neural responses. J. Neurosci. 29, 8525-8529.

Conflict of Interest Statement: The authors declare that the research was conducted in the absence of any commercial or financial relationships that could be construed as a potential conflict of interest.

Received: 26 January 2011; accepted: 12 May 2011; published online: 23 May 2011. Citation: Forgiarini $M$, Gallucci $M$ and Maravita A (2011) Racism and the empathy for pain on our skin. Front. Psychology 2:108. doi: 10.3389/fpsyg.2011.00108

This article was submitted to Frontiers in Perception Science, a specialty of Frontiers in Psychology.

Copyright ( $\odot 2011$ Forgiarini, Gallucci and Maravita. This is an open-access article subject to a non-exclusive license between the authors and Frontiers Media SA, which permits use, distribution and reproduction in other forums, provided the original authors and source are credited and other Frontiers conditions are complied with. 